\title{
ARRAY OF MICROMECHANICAL MASS SENSORS ENABLES HIGH-THROUGHPUT SINGLE-CELL GROWTH-RATE MEASUREMENTS
}

Selim Olcum, ${ }^{1}$ Nathan Cermak ${ }^{2}$ and Scott R. Manalis ${ }^{1,2}$

${ }^{1}$ Koch Institute for Integrative Cancer Research, ${ }^{2}$ Program in Computational and Systems Biology

Massachusetts Institute of Technology, Cambridge, MA, 02139 USA

\begin{abstract}
Technologies that rapidly assess cell growth would be transformative for many applications, including drug susceptibility testing for pathogens and cancer cells. Resonant micromechanical mass sensors can precisely measure growth, but are limited to measuring one cell at a time. Here, we demonstrate an approach to precisely and rapidly measure growth rates of many individual cells simultaneously. We flow cells in suspension through a microfluidic channel with multiple resonant mass sensors distributed along its length, weighing each cell repeatedly over the small fraction of a cell cycle it spends traversing this channel. Because multiple cells traverse this channel at the same time, we obtain growth rates up to 60 cells/h, while retaining a resolution of $0.2 \mathrm{pg} / \mathrm{h}$ for mammalian cells. Our system reveals subpopulations of cells with divergent growth kinetics and enables assessing cellular responses to antibiotics or antimicrobial peptides within minutes.
\end{abstract}

\section{INTRODUCTION}

Single cells are capable of remarkable variability across a range of behaviors, of which variation in growth is perhaps one of the most fundamental and important forms. From bacteria to unicellular eukaryotes to metazoan cells, even genetically identical cells may grow at drastically different rates, due to a combination of intrinsic molecular noise and various deterministic behavioral programs. This variation is typically not observable via populationbased growth assays, yet it has important consequences for human health.

Despite its importance, precisely and rapidly quantifying single-cell growth remains a technical hurdle. One approach is to measure a cell's outline in a microscopic image and then calculate its volume based on assumptions about its three-dimensional shape [1]. Another approach known as quantitative phase microscopy can be used to estimate a cell's dry mass over time [2]. Recently, inertial methods for measuring single-cell growth have been developed based on resonating micromechanical structures [3] that provide much higher precision than methods based on microscope imaging but are hindered by low throughput. Inertial methods exploit the fact that a micromechanical resonator's natural frequency depends on its mass. Adding cells to a resonator alters the resonator's mass and causes a measurable change in resonant frequency. One such class of resonator mass sensors is suspended microchannel resonators (SMRs), which consist of a sealed microfluidic channel that runs through the interior of a cantilever resonator. SMRs work by measuring the resonant frequency of a micro-cantilever suspended in vacuum, which is extremely sensitive to changes in mass. A feedback loop keeps the cantilever oscillating at its resonant frequency, while cells flow through a Ushaped microfluidic channel running the length of the cantilever. As a cell passes through the cantilever, the cantilever mass is transiently changed by the cell's buoyant mass (cell mass minus mass of the fluid it displaces), inducing a brief detectable change in the oscillation frequency. In water, a cell's buoyant mass is roughly proportional to (and is typically about one-quarter of) its dry mass [4]. SMRs are extremely precise, weighing single

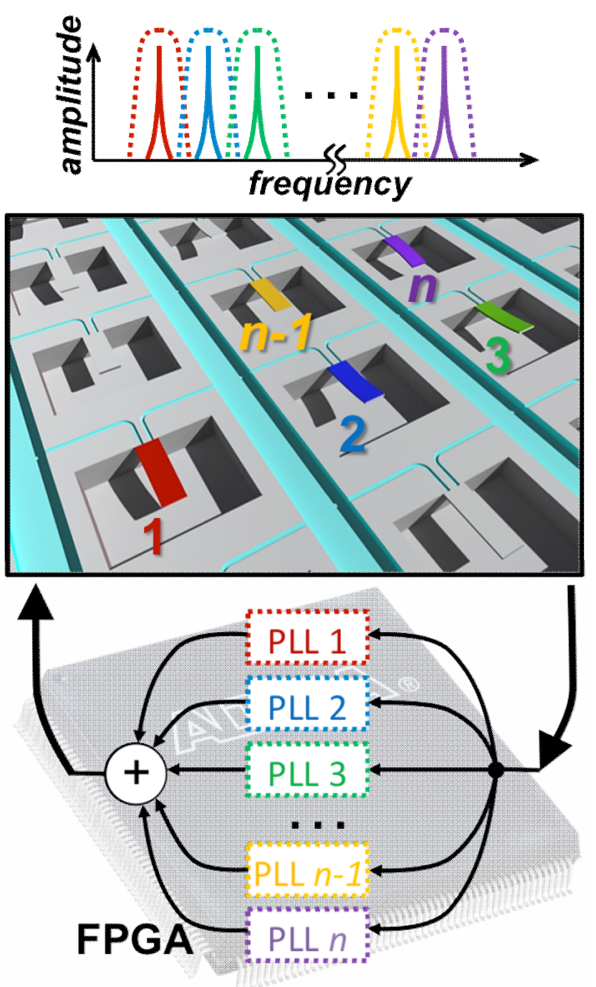

Figure 1: Independently and simultaneously oscillating a large array of mechanical resonators is challenging. A phase-locked loop (PLL) array implemented on a FPGA platform (bottom) can control individual resonators, if their resonances are separated in the frequency domain (top). This scalable approach requires carefully engineered resonator-PLL dynamics for the highest precision and minimum crosstalk.

mammalian cells with a resolution of $0.05 \mathrm{pg}(0.1 \%$ of a cell's buoyant mass) or better [3]. By flowing a cell back and forth through the cantilever repeatedly, SMRs can be used to measure a cell's growth rate, but are limited to measuring one cell at a time [5]. This limitation in throughput has prevented the wider application of SMRs across a range of biological and clinical efforts.

Here we introduce an SMR-based technique for highthroughput growth rate measurements that retains the SMR's mass and growth rate precision. We use an array of SMRs fluidically connected in series and separated by "delay" channels between each cantilever. These delay channels give the cell time to grow as it flows between cantilevers. After a cell exits a cantilever, other cells are free to enter it and be weighed. As a result, we are not limited to flowing only one cell through the array at a time, but can have many cells flowing in a queue. This enables high-throughput precision growth measurements across a wide array of suspended cell types.
Solid-State Sensors, Actuators and Microsystems Workshop Hilton Head Island, South Carolina, June 5-9, 2016 


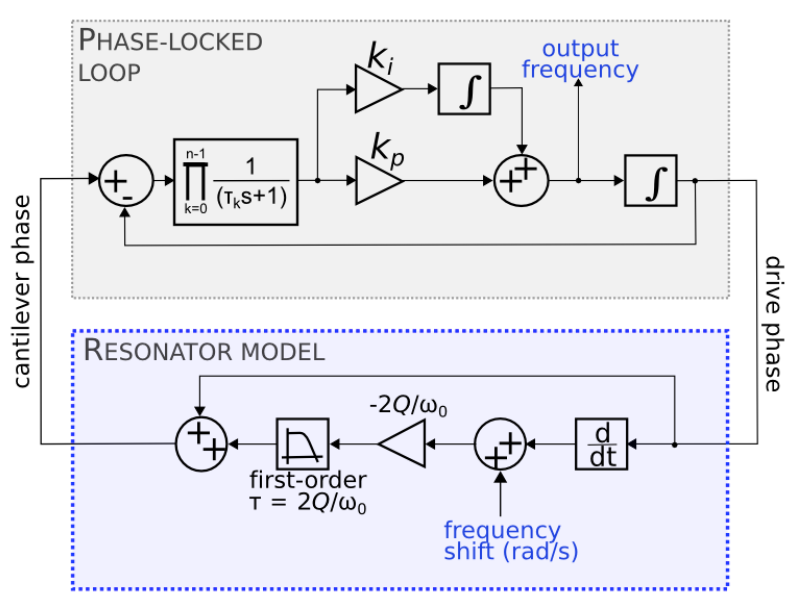

Figure 2: Linear phase-domain model of resonator-PLL system [6]. The PLL and the resonator are modelled at the top and the bottom boxes, respectively. We introduced additional poles at $\tau_{k}$ in the PLL model for achieving an overall $n^{\text {th }}$ order Butterworth type response (Table 1). No additional pole is required for a first-order response. $\tau$ in the resonator model is equal to $2 Q / \omega_{0}$, where $\omega_{0}$ and $Q$ are the resonant frequency and quality factor of the corresponding resonance.

\section{ARRAY OF MECHANICAL RESONATORS}

In order to retain the extreme precision of a single resonator in an array, each element must be oscillated independently. However it is challenging to isolate individual detection and actuation signal paths of the resonators due to fabrication and control complexities. To solve this problem, we design resonators with unique resonant frequencies (Figure 1, top, solid) and use a single physical actuation and a single physical detection channel. Therefore we can still independently actuate and detect each resonator individually (frequency division multiplexing) without the need of dedicated signal paths. We use an array of phaselocked loops (PLL) on an FPGA platform (Figure 1, bottom), where each PLL is assigned to control a single resonance [6]. We engineered the parameters of each PLL such that $i$. There is minimal crosstalk between the resonator channels and ii. Each PLL tracks the frequency of the corresponding resonator as closely as possible.

For the highest precision, we required resonator-PLL closedloop system for each mode has a transfer function identical to a Butterworth-type low-pass filter with controllable bandwidth. Therefore, we first derived the phase-domain transfer function of a harmonic oscillator around its resonant frequency [6] and used it to calculate the transfer function of a generic resonator-PLL system (Figure 2). Then, we showed by setting the PLL parameters (proportional gain, $k_{p}$, integral gain, $k_{i}$ and additional poles, $\tau_{k}$ ) using Table 1 that the resonator-PLL system simplifies to a Butterworth filter with desired bandwidth, $\beta$ and order, $n$. We used these findings on an array of PLLs implemented on a FPGA platform.

Table 1: PLL parameters for $n^{\text {th }}$ order Butterworth-type response with bandwidth, $\beta$.

\begin{tabular}{llcccc}
$n$ & $k_{p}$ & $k_{i}$ & $\tau_{1}$ & $\tau_{2}$ & Additional Poles \\
\hline 1 & $\beta$ & $k_{p} / \tau$ & & & 0 \\
2 & $\beta / \sqrt{2}$ & $k_{p} / \tau$ & $\sqrt{2} / 2 \beta$ & & 1 \\
3 & $\beta / 2$ & $k_{p} / \tau$ & $(1 \pm j) / 2 \beta$ & $(1 \mp j) / 2 \beta$ & 2 \\
\hline
\end{tabular}
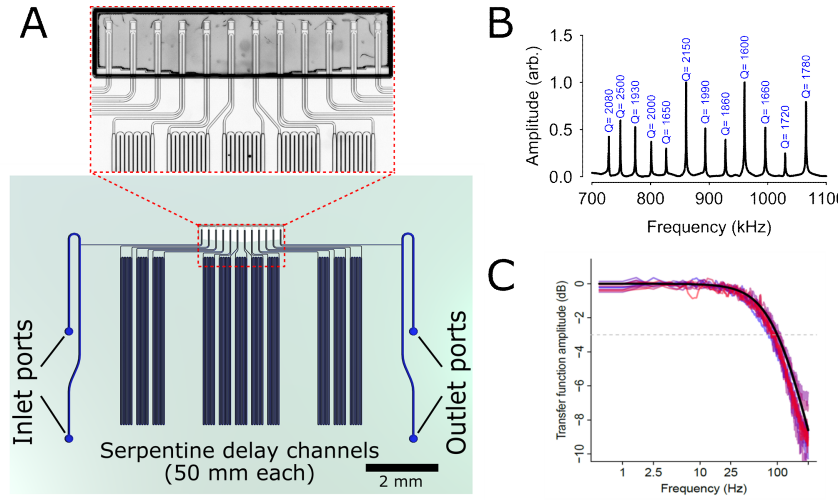

Figure 3: Schematic of the SMR array chip (A) along with an dark-field optical micrograph of the array of 12 cantilevers (inset) each with a unique resonant frequency (B). Each element in the PLL array is engineered such that the pass-bands of the resonator-PLL loops are flat, with a user-defined bandwidth $(C)$.

\section{SERIAL MICROCHANNEL RESONATOR ARRAYS}

We utilized the PLL array approach described in Figure 1 for oscillating twelve SMRs (Figure 3A) with uniformly-spaced resonant frequencies (Figure $3 \mathrm{~B}$ ). The frequencies and quality factors of each resonator in the array were used to set the corresponding PLL coefficients using Table 1 in order to achieve a first order response from the resonator-PLL control loops. Each PLL is configured such that it will track its cantilever's oscillation frequency with a bandwidth of $100 \mathrm{~Hz}$ (Figure 3C).

To simultaneously measure the vibrations of all cantilevers in the array, we employed an optical lever setup in which all cantilevers are simultaneously illuminated and a single photodetector measures the superposition of their deflection signals. The resulting deflection signal, which consists of the sum of 12 signals from the cantilever array, goes to the array of PLLs where each PLL locks to the unique resonant frequency of a single cantilever. Each PLL tracks its assigned cantilever's resonant frequency by demodulating its deflection signal and then generates a sinusoidal drive signal at that frequency. The drive signals from each PLL are then summed and used to drive a single piezo actuator positioned directly underneath the chip, completing the feedback loop.
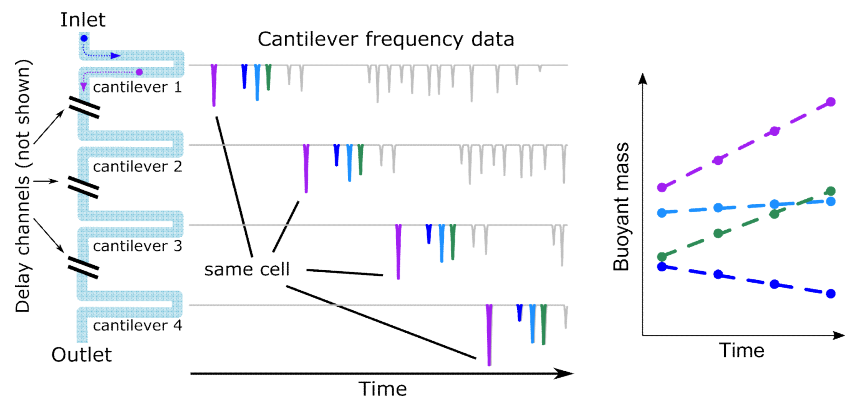

Figure 4: Simulated data showing frequency peaks originating from single cells flowing through a series of SMRs separated by delay channels. Cells grow as they traverse the array. After grouping frequency peaks originating from the same cell, that cell's growth rate can be obtained by regressing its buoyant mass versus time. Because many cells can traverse the array simultaneously, this device can achieve much higher throughput than a single sensor. 
After acquiring the frequency signals for each cantilever, we convert them to mass units via each cantilever's sensitivity (Hz/pg), which must be known precisely. Therefore, we measured the cantilever sensitivities during each experiment by spiking in inert monodisperse polystyrene particle size standards into all samples. Since the particles are highly uniform $(\sim 1 \%$ coefficient of variation in diameter), we can easily distinguish them even when they are of similar size to the cell sample of interest.

We utilized long serpentine "delay" channels between each SMR for giving the cells enough time to grow between mass measurements (Figure 3A). After a cell exits an SMR, other cells are free to enter it and get weighed. As a result, we are not limited
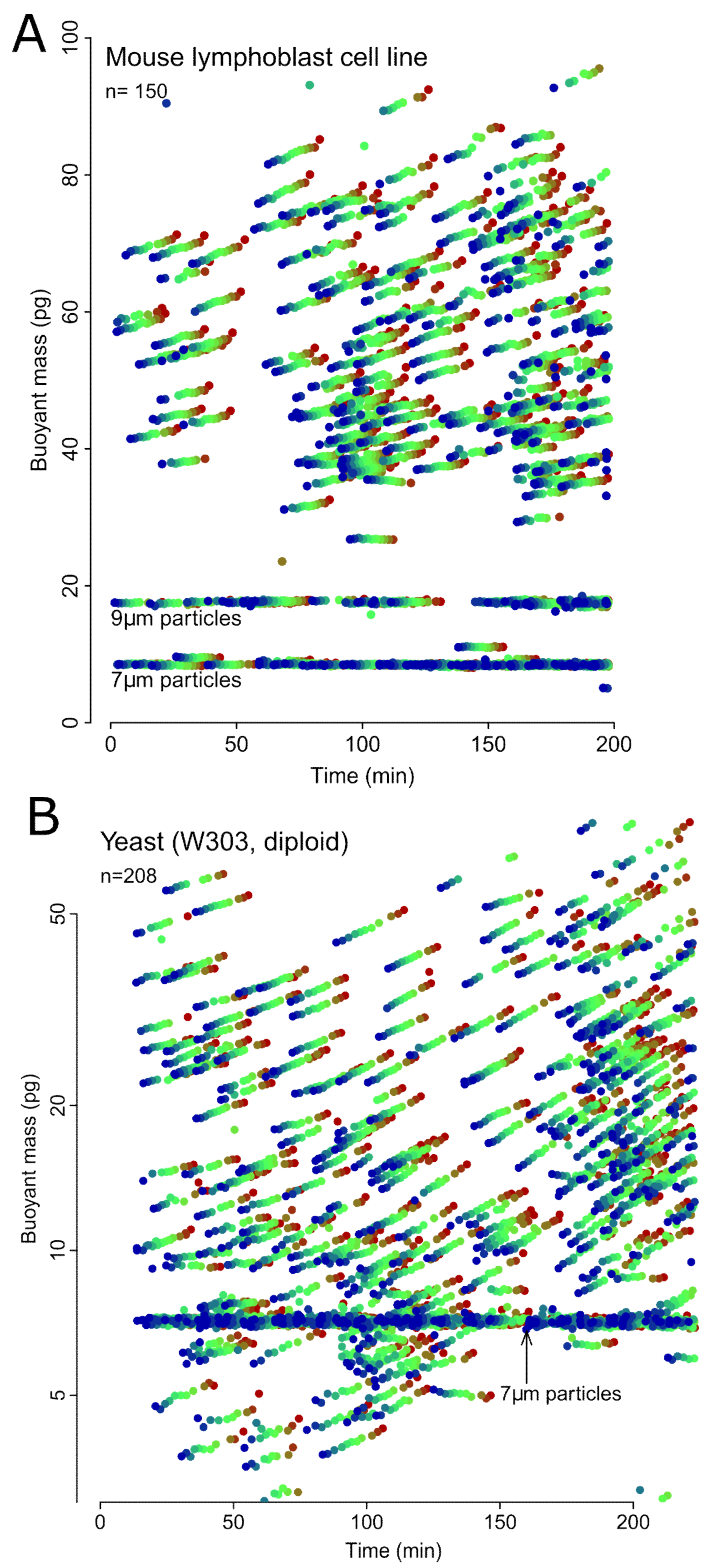

Figure 5: A) Growth data of lymphoid cell lines extracted from frequency shifts of cantilevers, color-coded by cantilever (blue is first cantilever, green is 6th cantilever, red is 12th cantilever). 7 and $9 \mu \mathrm{m}$ inert polystyrene particles are added as calibration and negative control, respectively. B) Lag-phase yeast culture growth dynamics in rich media measured at single-cell resolution. to flowing only one cell through the array at a time, but can have many cells flowing in a queue. This enables high-throughput precision growth measurements across a wide array of cell types. The time between measurements can be controlled by adjusting the flow rate. Therefore, we can easily tune the device's behavior to trade throughput for resolution by changing the flow rate, i.e. faster flow yields higher throughput but poorer resolution, and conversely for slower flow.

Here we demonstrate proof-of-concept SMR arrays to monitor the growth of steady-state L1210 cells (Fig. 5A), a mouse lymphoblast cell line previously studied with SMRs [7]. In a 200minute experiment, we measured the size and mass accumulation rate of 150 cells. In contrast, a single SMR measuring each cell for 20 minutes could measure $\leq 10$ cells as it takes some time to switch between cells. We next investigated whether serial SMR array could be used to study yeast ( $\mathrm{S}$. cerevisiae), a model organism of interest in biology. We measured the growth of single yeast cells in lag phase - a brief period of slow or no growth following transfer from spent media to fresh media. In a 3.5 hour experiment, we measured the mass accumulation rates of 208 cells (or cell clumps, as daughters often remain adhered to mothers following division), as shown in Fig. 5B. Here we've plotted these cells' mass on a logarithmic scale because single yeast cells grow exponentially. On a semilog plot, the slope of a cell's mass trajectory may be interpreted as an exponential growth rate, which is equivalent to its mass accumulation rate per unit mass.

\section{CONCLUSIONS}

We envision that this device will be applicable in many areas of research and potentially in clinical settings. The ability to detect rare growing cells in complex mixtures from patients could provide the possibility of correlating these measurements to disease states, and ultimately assessing drug susceptibility in rare cells. Yet the approach described here could be optimized further. Improving the actuation and detection schemes might enable an order of magnitude improvement in growth rate precision, and this extra precision can be exchanged directly for higher throughput by increasing the flow rates of cells transiting the array. Furthermore replacing the optical lever setup with embedded piezoresistive sensors could enable controlling hundreds of SMRs on a single chip.

\section{REFERENCES}

1. Aldridge, B. B. et al. Asymmetry and Aging of Mycobacterial Cells Lead to Variable Growth and Antibiotic Susceptibility. Science 335, 100-104 (2012).

2. Zangle, T. A. \& Teitell, M. A. Live-cell mass profiling: an emerging approach in quantitative biophysics. Nat. Methods 11, 1221-1228 (2014).

3. Burg, T. P. et al. Weighing of biomolecules, single cells and single nanoparticles in fluid. Nature 446, 1066-1069 (2007).

4. Feijó Delgado, F. et al. Intracellular water exchange for measuring the dry mass, water mass and changes in chemical composition of living cells. PloS One 8, e67590 (2013).

5. Godin, M. et al. Using buoyant mass to measure the growth of single cells. Nature Methods 7, 387-390 (2010).

6. Olcum, S., Cermak, N., Wasserman, S. C. \& Manalis, S. R. High-speed multiple-mode mass-sensing resolves dynamic nanoscale mass distributions. Nature Communications 6, (2015).

7. Son, S. et al. Direct observation of mammalian cell growth and size regulation. Nature Methods 9, 910-912 (2012). 